DOI: 10.12731/2070-7568-2020-1-184-198

УДК 004.94

\title{
ПРОГРАММНЫЙ КОМПЛЕКС \\ СКВОЗНОЙ АВТОМАТИЗАЦИИ ИМИТАЦИОННОГО МОДЕЛИРОВАНИЯ БИЗНЕС-ПРОЦЕССОВ
}

\section{Тюльпинова Н.В.}

Компьютерное моделирование в современных условиях становится основным инструментом повышения эффективности бизнеспроцессов благодаря его способности имитировать поведение реальных систем. Результативность такого моделирования зависит не только от структуры компьютерной модели - не менее важным является планирование, проведение и обработка имитационных экспериментов, так как отсутствие научно обоснованной программы вычислительного эксперимента может привести к получению некорректных результатов моделирования, расходящихся с реальностью. Однако, разработчики программного обеспечения для бизнессимуляции не уделяют должного вниманию этому вопросу: обзор функииональных возможностей инструментальных средств, ориентированных на моделирование, имитацию и анализ бизнес-процессов, показывает, что в настоящее время отсутствуют прикладные программные пакеты, реализующие сквозную автоматизациию ичикла «планирование - симуляция - обработка результатов».

Цель - разработка программного обеспечения, автоматизирующсего и интегрирующчего все этапы ичикла имитационного моделирования бизнес-прочессов.

Метод или методология проведения работы: математическая теория планирования экспериментов.

Результаты: программный комплекс и алгоритм проведения бизнес-симуляции в автоматическом режиме.

Область применения результатов: менеджмент (финансовый, стратегический, антикризисный, инвестиционный, инноваизионный, риск-менеджмент). 
Ключевые слова: имитационное моделирование; планирование экспериментов; бизнес-процесс.

\section{SOFTWARE PACKAGE FOR END-TO-END AUTOMATION SIMULATION OF BUSINESS PROCESSES}

\section{Tyulpinova N.V.}

In the modern conditions computer simulation becomes an essential tool improving the efficiency of business processes, due its ability to mimic the behavior of the real systems. The effectiveness of the simulation depends not only on the model design-planning, implementation and processing of simulation experiments are equally important, because the absence of scientifically based program for the computational experiment causes incorrect results, disagreement to reality. However, developers of the business-simulation software do not pay due attention to this aspect: the overview of the functional of applications for modeling, simulation and analysis of business processes shows, that nowadays there are no applied software packages, which accomplish end-to-end automation for cycle "planning - simulation - processing».

Purpose-development the software for automation and integration of the whole simulation cycle of business processes.

Method or methodology of the work: mathematical methods for design of experiments.

Results: the software package and the algorithm for business simulations by automatic way.

Practical implications: management (financial, strategic, crisis, investment, innovative, risk).

Keywords: simulation modeling; design of experiments; business process.

Выявление и всестороннее исследование зависимостей и взаимосвязей между объективно существующими явлениями бизнеспроцессов является чрезвычайно важным, так как даёт возможность 
раскрыть механизм причинно-следственных отношений между происходящими событиями. При этом вербальное описание этих объективно существующих зависимостей и взаимосвязей весьма не информативно, так как на практике требуется количественное описание тесноты причинно-следственных связей и формы влияний. Для исследования интенсивности, вида и формы причинных влияний незаменимым является имитационное моделирование в сочетании с планированием экспериментов. В приложении к бизнес-процессам это тот инструмент, который позволяет вскрыть сложные комплексы причин и следствий, сформировать векторы совершенствования существующих бизнес-практик, виртуально протестировать новые идеи, после чего принять обоснованное бизнес-решение. Выявление количественных соотношений в виде математических зависимостей позволяет лучше понять природу исследуемого явления, что в свою очередь открывает возможность целенаправленно воздействовать на выявленные факторы, вмешиваться в соответствующий бизнеспроцесс с целью получения требуемых результатов.

Компьютерное моделирование в настоящее время становится основным инструментом повышения эффективности бизнес-процессов благодаря его способности имитировать поведение реальных систем. Однако при его практическом применении возникает далеко не тривиальный вопрос: какие именно и в каком количестве конфигурации бизнес-процессов следует смоделировать, чтобы получить требуемую информацию, поскольку если последовательность имитационных прогонов выполняется бессистемно (т.е. наугад проверяется ряд альтернативных конфигураций и наблюдается, что при них происходит), то это ведёт к получению случайных, не объективных и не достоверных результатов. Более того, если на исследуемый бизнес-процесс в действительности воздействует не один, а множество факторов и межфакторных взаимодействий с различной степенью и интенсивностью влияния, то не спланированная на научной основе серия имитационных экспериментов в лучшем случае всего лишь не позволит выявить указанное воздействие, а в худшем - приведёт к получению некорректных ре- 
зультатов моделирования, расходящихся с реальностью. Не менее важным в имитационном моделировании бизнес-процессов является и тот факт, что информативный результат должен быть получен при наименьшем объеме затрачиваемых вычислительных ресурсов и минимальном объеме машинного (процессорного) времени. Для решения обозначенных проблем в основу имитационного моделирования должна быть положена математическая теория планирования экспериментов [11-15], которая сделает процесс симуляции целенаправленным и организованным, что существенно повысит как надежность получаемых результатов, так и эффективность имитационных экспериментов, при этом совокупность операций планирования, выполнения и обработки результатов имитационных экспериментов должна быть полностью интегрирована и переведена в автоматический режим, а исследователь-пользователь (например, риск-менеджер, финансовый менеджер, инвестиционный менеджер, системный аналитик и др.), в зависимости от заданных им исследуемых факторов, должен оперативно получать результат моделирования в виде математической зависимости, описывающей исследуемый бизнес-процесс с целью его последующей оптимизации. Обзор функциональных возможностей программных средств для имитационного моделирования [1-10] показывает, что в настоящее время отсутствуют прикладные программные пакеты, реализующие сквозную автоматизацию цикла «планирование - симуляция - обработка результатов». В этой связи, представленные ниже результаты исследований - разработанное алгоритмическое и программное обеспечение для автоматизации и интеграции всех этапов цикла имитационного моделирования - представляют интерес как с научной, так и с практической точки зрения.

Алгоритм проведения вычислительного эксперимента с использованием разработанного программного комплекса включает следующие этапы.

Этап №1. Ввод исходных данных (рис. 1а). На данном этапе необходимо вначале задать число исследуемых факторов и число повторений каждого опыта (в качестве математического ядра 
запрограммирован алгоритм двухуровневого полного факторного эксперимента [11-15]); затем нажать кнопку «Ввод» и в появившейся таблице ввести числовые значения уровней и интервала варьирования каждого фактора (число столбцов таблицы динамически изменяется в зависимости от количества исследуемых факторов); после чего нажать кнопку «Сформировать план и выполнить имитационный эксперимент».

Этап №2. Планирование имитационного эксперимента. По итогам этапа №1 автоматически генерируется и записывается в файл рандомизованный сценарий эксперимента (рис. 1б), содержащий последовательность реализации опытов и числовые значения факторов для каждого опыта.

Этап №3. Выполнение имитационного эксперимента. Файл, сформированный по итогам этапа №2, считывается средой имитационного моделирования, которая в режиме пакетной обработки данных выполняет пакет заданий файла, т.е. автоматически реализует серию имитационных экспериментов в порядке, заданном файлом этапа №2 (формат этого файла поддерживается подавляющим большинством современных систем имитационного моделирования). В процессе реализации этапа №3 последовательно, в автоматическом режиме, формируется файл результатов серии экспериментов (рис. 1в).

Этап №4. Представление результатов в соответствии с основными положениями теории планирования эксперимента (рис. 1г): табличная систематизация содержимого файлов сценария и результатов серии экспериментов. После нажатия кнопки «Обработать результаты имитационного эксперимента», запускается процедура автоматической статистической обработки результатов эксперимента, включающая в себя этапы №5, №6, №7, №8 (на каждом из этих этапов система запрашивает у пользователя требуемый уровень статистической значимости).

Этап №5. Проверка однородности дисперсий по критерию Кохрена (рис. 2a), в ходе которой рассчитываются следующие показатели: 1) среднее значе ние исследуемого параметра по реализации 
a)

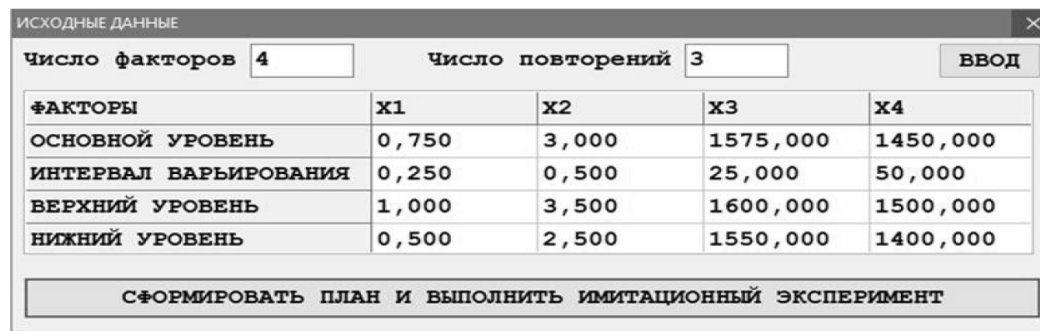

6)

\begin{tabular}{|c|c|}
\hline $\begin{array}{l}\text { test } \\
\text { x1 } \\
\text { x2 } \\
\text { x3 } \\
\text { x4 }\end{array}$ & $\begin{array}{l}1(10) \\
0.500 \\
3.500 \\
1600.000 \\
1400.000\end{array}$ \\
\hline $\begin{array}{l}\text { test } \\
\text { x1 } \\
\times 2 \\
\times 3 \\
\text { x } \vdots \\
\text { 4 }\end{array}$ & $\begin{array}{l}2(14) \\
0.500 \\
3.500 \\
1550.000 \\
1400.000\end{array}$ \\
\hline $\begin{array}{l}\text { test } \\
\text { X1: } \\
\text { x2 } \\
\times 3: \\
\text { x } 4:\end{array}$ & $\begin{array}{l}3(4) \\
0.500 \\
2.500 \\
1600.000 \\
1500.000\end{array}$ \\
\hline $\begin{array}{l}\text { test } \\
\times 1 \vdots \\
\times 2 \vdots \\
\times 3 \vdots \\
\times 4:\end{array}$ & $\begin{array}{l}4(3) \\
1.000 \\
2.500 \\
1600.000 \\
1500.000\end{array}$ \\
\hline $\begin{array}{l}\text { test } \\
\text { x1: } \\
\text { x2: } \\
\text { x3: } \\
\text { x4: }\end{array}$ & $\begin{array}{l}5(7) \\
1.000 \\
2.500 \\
1550.000 \\
1500.000\end{array}$ \\
\hline $\begin{array}{l}\text { test } \\
\text { x1 } \\
\times 2 \vdots \\
\times 3 \vdots \\
\text { x4 }\end{array}$ & $\begin{array}{l}6(8) \\
0.500 \\
2.500 \\
1550.000 \\
1500.000\end{array}$ \\
\hline $\begin{array}{l}\text { test } \\
\times 1: \\
\times 2: \\
\times 3: \\
\times 4:\end{array}$ & $\begin{array}{l}7(2) \\
0.500 \\
3.500 \\
1600.000 \\
1500.000\end{array}$ \\
\hline $\begin{array}{l}\text { test } \\
\text { X1 } \\
\times 2 \\
\times 3 \\
\times 4 \\
\text { x4 }\end{array}$ & $\begin{array}{l}8(9) \\
1.000 \\
3.500 \\
1600.000 \\
1400.000\end{array}$ \\
\hline $\begin{array}{l}\text { test } \\
\times 1: \\
\times 2: \\
\times 3: \\
\times 4 \vdots\end{array}$ & $\begin{array}{l}9(15) \\
1.000 \\
2.500 \\
1550.000 \\
1400.000\end{array}$ \\
\hline $\begin{array}{l}\text { test } \\
\times 1: \\
\times 22 \\
\times 3: \\
\times 4:\end{array}$ & $\begin{array}{l}10(13) \\
1.000 \\
3.500 \\
1550.000 \\
1400.000\end{array}$ \\
\hline $\begin{array}{l}\text { test } \\
\times 1: \\
\times 2 \vdots \\
\times 3 \vdots \\
\times 4 \vdots\end{array}$ & $\begin{array}{l}11(16) \\
0.500 \\
2.500 \\
1550.000 \\
1400.000\end{array}$ \\
\hline $\begin{array}{l}\text { test } \\
\text { x1: } \\
\times 2 \vdots \\
\times 3 \vdots \\
\times 4:\end{array}$ & $\begin{array}{l}12(6) \\
0.500 \\
3.500 \\
1550.000 \\
1500.000\end{array}$ \\
\hline
\end{tabular}

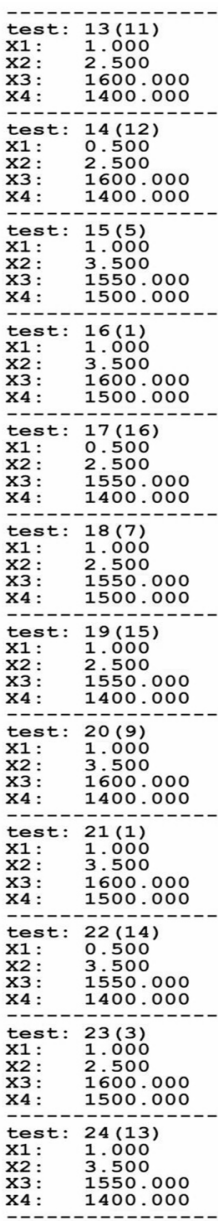

\begin{tabular}{|c|c|}
\hline $\begin{array}{l}\text { test: } \\
\text { x1 } \\
\times 22 \\
\times 3 \\
\times 3 \\
\times 4\end{array}$ & $\begin{array}{l}37(11) \\
1.000 \\
2.500 \\
1600.000 \\
1400.000\end{array}$ \\
\hline $\begin{array}{l}\text { test: } \\
\times 1: \\
\times 2 \vdots \\
\times 3 \vdots \\
\times 4 \vdots\end{array}$ & $\begin{array}{l}38(7) \\
1.000 \\
2.500 \\
1550.000 \\
1500.000\end{array}$ \\
\hline $\begin{array}{l}\text { test: } \\
\text { x1 } \\
\times 22 \\
\times 33 \\
\times 4 \\
\text { x4 }\end{array}$ & $\begin{array}{l}39(15) \\
1.000 \\
2.500 \\
1550.000 \\
1400.000\end{array}$ \\
\hline $\begin{array}{l}\text { test: } \\
\text { test } \\
\times 22 \\
\times 3 \vdots \\
\times 4 \vdots \\
\times 4\end{array}$ & $\begin{array}{l}40(8) \\
0.500 \\
2.500 \\
1550.000 \\
1500.000\end{array}$ \\
\hline $\begin{array}{l}\text { test: } \\
\text { test } \\
\times 22 \\
\times 3 \vdots \\
\times 4 \vdots\end{array}$ & $\begin{array}{l}41(5) \\
1.000 \\
3.500 \\
1550.000 \\
1500.000\end{array}$ \\
\hline $\begin{array}{l}\text { test: } \\
\times 1 \vdots \\
\times 22 \\
\times 3 \vdots \\
\times 4 \vdots\end{array}$ & $\begin{array}{l}42(12) \\
0.500 \\
2.500 \\
1600.000 \\
1400.000\end{array}$ \\
\hline $\begin{array}{l}\text { test: } \\
\times 1: \\
\times 20 \\
\times 3 \vdots \\
\times 4:\end{array}$ & $\begin{array}{l}43(14) \\
0.500 \\
3.500 \\
1550.000 \\
1400.000\end{array}$ \\
\hline $\begin{array}{l}\text { test: } \\
\text { x1 } \\
\times 22 \\
\times 3 \vdots \\
\times 4 \vdots\end{array}$ & $\begin{array}{l}44(3) \\
1.000 \\
2.500 \\
1600.000 \\
1500.000\end{array}$ \\
\hline $\begin{array}{l}\text { test: } \\
\text { test: } \\
\times 12 \\
\times 3 \\
\times 3 \vdots \\
\times 4 !\end{array}$ & $\begin{array}{l}45(4) \\
0.500 \\
2.500 \\
1600.000 \\
1500.000\end{array}$ \\
\hline $\begin{array}{l}\text { test: } \\
\text { test } \\
\times 22 \\
\times 3 \vdots \\
\times 4 \vdots\end{array}$ & $\begin{array}{l}46(6) \\
0.500 \\
3.500 \\
1550.000 \\
1500.000\end{array}$ \\
\hline $\begin{array}{l}\text { test: } \\
\text { test } \\
\times 2 \vdots \\
\times 3 \vdots \\
\times 4 \vdots \\
\times 4\end{array}$ & $\begin{array}{l}47(16) \\
0.500 \\
2.500 \\
1550.000 \\
1400.000\end{array}$ \\
\hline $\begin{array}{l}\text { test: } \\
\text { test } \\
\times 12 \\
\times 3 \\
\times 3 \\
\times 4\end{array}$ & $\begin{array}{l}48(9) \\
1.000 \\
3.500 \\
1600.000 \\
1400.000\end{array}$ \\
\hline
\end{tabular}

Рис. 1. Планирование и выполнение имитационного эксперимента (начало) 
B)

\begin{tabular}{|c|c|}
\hline $\begin{array}{l}\text { result: } \\
\text { Y: }\end{array}$ & $\begin{array}{l}1(10) \\
655\end{array}$ \\
\hline $\begin{array}{l}\text { result: } \\
\text { Y: }\end{array}$ & $\begin{array}{l}2(14) \\
635\end{array}$ \\
\hline $\begin{array}{l}\text { result: } \\
Y:\end{array}$ & $\begin{array}{l}3(4) \\
565\end{array}$ \\
\hline $\begin{array}{l}\text { result: } \\
\text { Y: }\end{array}$ & $\begin{array}{l}4(3) \\
640\end{array}$ \\
\hline $\begin{array}{l}\text { result: } \\
Y:\end{array}$ & $\begin{array}{l}5(7) \\
610\end{array}$ \\
\hline $\begin{array}{l}\text { result: } \\
Y:\end{array}$ & $\begin{array}{l}6(8) \\
500\end{array}$ \\
\hline $\begin{array}{l}\text { result: } \\
Y:\end{array}$ & $\begin{array}{l}7(2) \\
785\end{array}$ \\
\hline $\begin{array}{l}\text { result: } \\
\text { Y: }\end{array}$ & $\begin{array}{l}8(9) \\
410\end{array}$ \\
\hline $\begin{array}{l}\text { result: } \\
\text { Y: }\end{array}$ & $\begin{array}{l}9(15) \\
290\end{array}$ \\
\hline $\begin{array}{l}\text { result: } \\
\text { Y: }\end{array}$ & $\begin{array}{l}10(13) \\
405\end{array}$ \\
\hline $\begin{array}{l}\text { result: } \\
Y:\end{array}$ & $11(16)$ \\
\hline $\begin{array}{l}\text { result: } \\
\text { Y: }\end{array}$ & $\begin{array}{l}12(6) \\
765\end{array}$ \\
\hline
\end{tabular}

\begin{tabular}{|c|c|}
\hline $\begin{array}{l}\text { result: } \\
\text { Y: }\end{array}$ & $\begin{array}{l}13(11) \\
385\end{array}$ \\
\hline $\begin{array}{l}\text { result: } \\
\mathrm{Y}:\end{array}$ & $\begin{array}{l}14(12) \\
350\end{array}$ \\
\hline $\begin{array}{l}\text { result: } \\
Y:\end{array}$ & $\begin{array}{l}15(5) \\
895\end{array}$ \\
\hline $\begin{array}{l}\text { result: } \\
Y:\end{array}$ & $\begin{array}{l}16(1) \\
990\end{array}$ \\
\hline $\begin{array}{l}\text { result: } \\
\text { Y: }\end{array}$ & $\begin{array}{l}17(16) \\
320\end{array}$ \\
\hline $\begin{array}{l}\text { result: } \\
\text { Y: }\end{array}$ & $\begin{array}{l}18(7) \\
590\end{array}$ \\
\hline $\begin{array}{l}\text { result: } \\
Y \text { : }\end{array}$ & $\begin{array}{l}19(15) \\
315\end{array}$ \\
\hline $\begin{array}{l}\text { result: } \\
\mathrm{Y}:\end{array}$ & $\begin{array}{l}20(9) \\
420\end{array}$ \\
\hline $\begin{array}{l}\text { result: } \\
Y \text { : }\end{array}$ & $\begin{array}{l}21(1) \\
960\end{array}$ \\
\hline $\begin{array}{l}\text { result: } \\
\mathbf{Y}:\end{array}$ & $\begin{array}{l}22(14) \\
615\end{array}$ \\
\hline $\begin{array}{l}\text { result: } \\
Y \text { : }\end{array}$ & $\begin{array}{l}23(3) \\
650\end{array}$ \\
\hline $\begin{array}{l}\text { result: } \\
Y:\end{array}$ & $\begin{array}{l}24(13) \\
375\end{array}$ \\
\hline
\end{tabular}

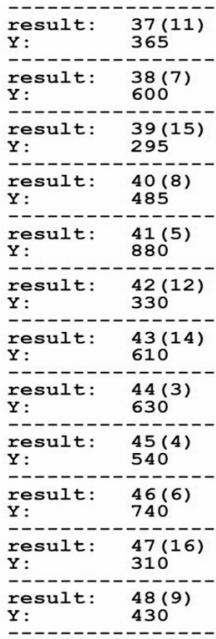

Г)

\begin{tabular}{|c|c|c|c|c|c|c|c|c|c|c|c|c|c|c|}
\hline \multirow{2}{*}{\begin{tabular}{|l|} 
номер \\
опыта
\end{tabular}} & \multicolumn{3}{|c|}{ Рандомизация } & \multicolumn{4}{|c|}{ Кодовые значения } & \multicolumn{4}{|c|}{ натуральные значения } & \multicolumn{3}{|c|}{ Результаты } \\
\hline & 1 & 2 & 3 & x1 & x2 & x3 & $\mathrm{X} 4$ & x1 & $\mathrm{x} 2$ & $\times 3$ & $\times 4$ & Y1 & Y2 & Y3 \\
\hline 1 & 10 & 16 & 2 & +1 & +1 & +1 & +1 & 1,000 & 3,500 & 1600,000 & 1500,000 & 990 & 960 & 960 \\
\hline 2 & 14 & 7 & 13 & -1 & +1 & +1 & +1 & 0,500 & 3,500 & 1600,000 & 1500,000 & 785 & 810 & 805 \\
\hline 3 & 4 & 15 & 10 & +1 & -1 & +1 & +1 & 1,000 & 2,500 & 1600,000 & 1500,000 & 640 & 650 & 630 \\
\hline 4 & 3 & 9 & 1 & -1 & -1 & +1 & +1 & 0,500 & 2,500 & 1600,000 & 1500,000 & 565 & 545 & 540 \\
\hline 5 & 7 & 1 & 11 & +1 & +1 & -1 & +1 & 1,000 & 3,500 & 1550,000 & 1500,000 & 895 & 875 & 880 \\
\hline 6 & 8 & 14 & 7 & -1 & +1 & -1 & +1 & 0,500 & 3,500 & 1550,000 & 1500,000 & 765 & 745 & 740 \\
\hline 7 & 2 & 3 & 15 & +1 & -1 & -1 & +1 & 1,000 & 2,500 & 1550,000 & 1500,000 & 610 & 590 & 600 \\
\hline 8 & 9 & 13 & 8 & -1 & -1 & -1 & +1 & 0,500 & 2,500 & 1550,000 & 1500,000 & 500 & 515 & 485 \\
\hline 9 & 15 & 2 & 5 & +1 & +1 & +1 & -1 & 1,000 & 3,500 & 1600,000 & 1400,000 & 410 & 420 & 430 \\
\hline 10 & 13 & 11 & 12 & -1 & +1 & +1 & -1 & 0,500 & 3,500 & 1600,000 & 1400,000 & 655 & 695 & 690 \\
\hline 11 & 16 & 6 & 14 & +1 & -1 & +1 & -1 & 1,000 & 2,500 & 1600,000 & 1400,000 & 385 & 390 & 365 \\
\hline 12 & 6 & 5 & 3 & -1 & -1 & +1 & -1 & 0,500 & 2,500 & 1600,000 & 1400,000 & 350 & 340 & 330 \\
\hline 13 & 11 & 8 & 4 & +1 & +1 & -1 & -1 & 1,000 & 3,500 & 1550,000 & 1400,000 & 405 & 375 & 390 \\
\hline 14 & 12 & 10 & 6 & -1 & +1 & -1 & -1 & 0,500 & 3,500 & 1550,000 & 1400,000 & 635 & 615 & 610 \\
\hline 15 & 5 & 12 & 16 & +1 & -1 & -1 & -1 & 1,000 & 2,500 & 1550,000 & 1400,000 & 290 & 315 & 295 \\
\hline 16 & 1 & 4 & 9 & -1 & -1 & -1 & -1 & 0,500 & 2,500 & 1550,000 & 1400,000 & 330 & 320 & 310 \\
\hline & & & & & ab & NIt & REW & TATbl & ЦИона & ЭКСПЕРИ & HTA & & & \\
\hline
\end{tabular}

Окончание рис. 1.

серии параллельных опытов; 2) дисперсии отклонений исследуемого параметра от среднего значения; 3) сумма всех дисперсий; 4) максимальная дисперсия; 5) расчетное значение критерия Кохрена как отношение максимальной дисперсии к сумме всех дисперсий; 6) табличное значение критерия Кохрена, соответствующее числу степеней свободы максимальной дисперсии и числу степеней свободы суммы всех дисперсий при заданном уровне значимости. Итогом данного этапа яв- 
ляется вывод об однородности или неоднородности дисперсий: если расчетное значение критерия Кохрена оказывается меньше табличного значения этого критерия, то гипотеза об однородности дисперсий и воспроизводимости результатов принимается, а на экране появляется вывод «Дисперсии однородны»; в противном случае, если проверка дает отрицательный результат, - на экране появляется вывод о неоднородности дисперсий и рекомендация относительно увеличения числа параллельных опытов и проведения новой серии экспериментов.

Этап №6. Расчет коэффициентов уравнения регрессии (рис. 2б): каждый коэффициент представляет собой алгебраическую сумму произведений двух столбцов: фактора (или межфакторного взаимодействия) в кодовых обозначениях и среднего значения исследуемого параметра по реализации серии параллельных опытов, деленную на число точек в плане эксперимента.

Этап №7. Проверка значимости коэффициентов уравнения регрессии по критерию Стьюдента (рис. 2в), в ходе которой рассчитываются следующие показатели: 1) среднее арифметическое всех дисперсий; 2) дисперсия коэффициентов регрессии; 3) среднеквадратическое отклонение дисперсии коэффициентов регрессии; 4) расчетное значение критерия Стьюдента как отношение модуля коэффициента регрессии к среднеквадратическому отклонению дисперсии коэффициента регрессии; 5) табличное значение критерия Стьюдента, соответствующее числу степеней свободы для проверки значимости коэффициентов регрессии при заданном уровне значимости. Итогом данного этапа является вывод о значимости или незначимости коэффициентов регрессии: если расчетное значение критерия Стьюдента оказывается больше табличного значения этого критерия, то коэффициент признается значимым и на экране напротив данного коэффициента появляется вывод «Значим», в противном случае коэффициент считается статистически незначимым, ему соответствует вывод «Нет» (такой коэффициент отбрасывается без пересчета остальных коэффициентов), а если все коэффициенты незначимы, тогда на экране появляется рекомендация относительно увеличения интервалов варьирования факторов и проведения новой серии экспериментов. 


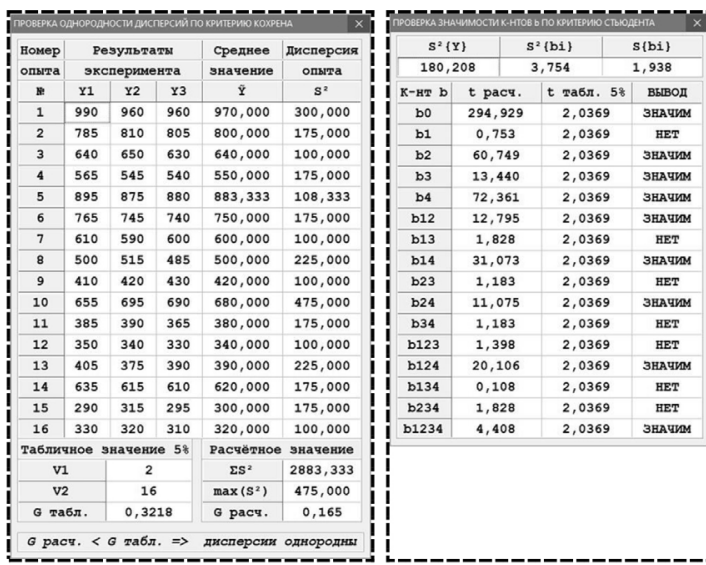

a)
B)

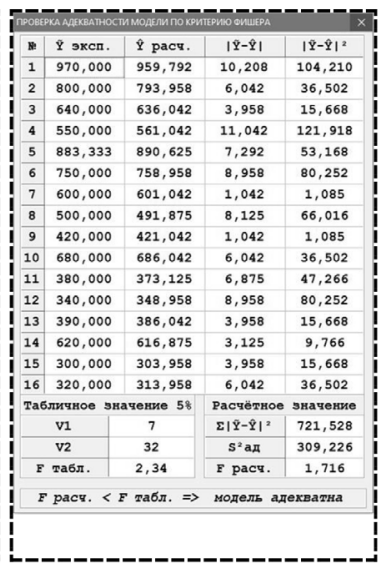

г)

\begin{tabular}{|c|c|c|c|c|c|c|c|c|c|c|c|c|c|c|c|c|c|}
\hline \multirow{2}{*}{$\begin{array}{l}\text { номер } \\
\text { опыта }\end{array}$} & \multicolumn{5}{|c|}{ факторы } & \multicolumn{11}{|c|}{ Взаимодействия факторов } & \multirow{2}{*}{\begin{tabular}{|c} 
Результат \\
$\tilde{Y}$
\end{tabular}} \\
\hline & 0 & 1 & 2 & 3 & 4 & 12 & 13 & 14 & 23 & 24 & 34 & 123 & 124 & 134 & 234 & 1234 & \\
\hline 1 & +1 & +1 & +1 & +1 & +1 & +1 & +1 & +1 & +1 & +1 & +1 & +1 & +1 & +1 & +1 & +1 & 970,000 \\
\hline 2 & +1 & -1 & +1 & +1 & +1 & -1 & -1 & -1 & +1 & +1 & +1 & -1 & -1 & -1 & +1 & -1 & 800,000 \\
\hline 3 & +1 & +1 & -1 & +1 & +1 & -1 & +1 & +1 & -1 & -1 & +1 & -1 & -1 & +1 & -1 & -1 & 640,000 \\
\hline 4 & +1 & -1 & -1 & +1 & +1 & +1 & -1 & -1 & -1 & -1 & +1 & +1 & +1 & -1 & -1 & +1 & 550,000 \\
\hline 5 & +1 & +1 & +1 & -1 & +1 & +1 & -1 & +1 & -1 & +1 & -1 & -1 & +1 & -1 & -1 & -1 & 883,333 \\
\hline 6 & +1 & -1 & +1 & -1 & +1 & -1 & +1 & -1 & -1 & +1 & -1 & +1 & -1 & +1 & -1 & +1 & 750,000 \\
\hline 7 & +1 & +1 & -1 & -1 & +1 & -1 & -1 & +1 & +1 & -1 & -1 & +1 & -1 & -1 & +1 & +1 & 600,000 \\
\hline 8 & +1 & -1 & -1 & -1 & +1 & +1 & +1 & -1 & +1 & -1 & -1 & -1 & +1 & +1 & +1 & -1 & 500,000 \\
\hline 9 & +1 & +1 & +1 & +1 & -1 & +1 & +1 & -1 & +1 & -1 & -1 & +1 & -1 & -1 & -1 & -1 & 420,000 \\
\hline 10 & +1 & -1 & +1 & +1 & -1 & -1 & -1 & +1 & +1 & -1 & -1 & -1 & +1 & +1 & -1 & +1 & 680,000 \\
\hline 11 & +1 & +1 & -1 & +1 & -1 & -1 & +1 & -1 & -1 & +1 & -1 & -1 & +1 & -1 & +1 & +1 & 380,000 \\
\hline 12 & +1 & -1 & -1 & +1 & -1 & +1 & -1 & +1 & -1 & +1 & -1 & +1 & -1 & +1 & +1 & -1 & 340,000 \\
\hline 13 & +1 & +1 & +1 & -1 & -1 & +1 & -1 & -1 & -1 & -1 & +1 & -1 & -1 & +1 & +1 & +1 & 390,000 \\
\hline 14 & +1 & -1 & +1 & -1 & -1 & -1 & +1 & +1 & -1 & -1 & +1 & +1 & +1 & -1 & +1 & -1 & 620,000 \\
\hline 15 & +1 & +1 & -1 & -1 & -1 & -1 & -1 & -1 & +1 & +1 & +1 & +1 & +1 & +1 & -1 & -1 & 300,000 \\
\hline 16 & +1 & -1 & -1 & -1 & -1 & +1 & +1 & +1 & +1 & +1 & +1 & -1 & -1 & -1 & -1 & +1 & 320,000 \\
\hline & 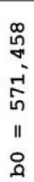 & 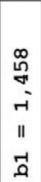 & 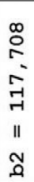 & 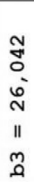 & 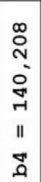 & 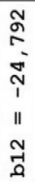 & 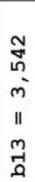 & 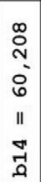 & 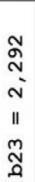 & 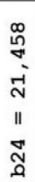 & 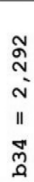 & 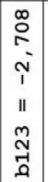 & 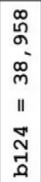 & 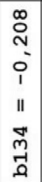 & 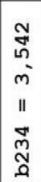 & 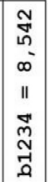 & \\
\hline
\end{tabular}

б)

Рис. 2. Статистическая обработка результатов эксперимента

Этап №8. Проверка адекватности модели по критерию Фишера (рис. 2г), в ходе которой рассчитываются следующие показатели: 1) экспериментальное значение исследуемого параметра; 2) рас- 
четное значение исследуемого параметра по уравнению регрессии; 3) модуль и квадрат разности между экспериментальным и расчетным значениями; 4) сумма полученных квадратов разностей; 5) дисперсия адекватности; 6) расчетное значение критерия Фишера как отношение дисперсии адекватности к дисперсии исследуемого параметра; 7) табличное значение критерия Фишера, соответствующее числу степеней свободы для дисперсии адекватности и числу степеней свободы для дисперсии исследуемого параметра при заданном уровне значимости. Итогом данного этапа является вывод об адекватности или неадекватности модели: если расчетное значение критерия Фишера оказывается меньше табличного значения этого критерия, то гипотеза адекватности модели принимается, а на экране появляется вывод «Модель адекватна»; в противном случае, если проверка дает отрицательный результат, - на экране появляется вывод о неадекватности модели и рекомендация относительно либо увеличения интервалов варьирования факторов; либо выделения и фиксации на определенном уровне фактора, порождающего неадекватность; либо преобразования факторов. a) $\begin{aligned} \mathrm{Y}=(\overline{57} \overline{1}, 458)+(11 \overline{7}, 708) \times 2+(2 \overline{6}, 042) \times 3+(140, \overline{208}) \times 4+(-\overline{4}, \overline{79}) \times 1 \times 2 \\ +\end{aligned}$

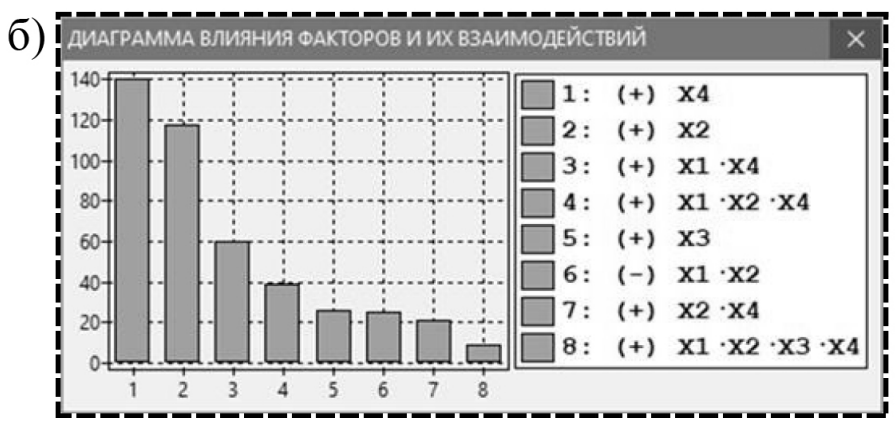

Рис. 3. Результаты имитационного моделирования

Этап №9. Автоматическая генерация результатов: 1) полученная по итогам моделирования регрессионная зависимость, описывающая исследуемый бизнес-процесс (рис. 3a); 2) диаграмма влияния 
факторов и межфакторных взаимодействий на параметры анализируемого бизнес-процесса (рис. 3б).

Разработанный программный комплекс полностью автоматизирует весь цикл имитационного моделирования от планирования и симуляции до обработки результатов эксперимента: пользователю достаточно лишь ввести исходные данные, после чего в автоматическом режиме (без участия человека) осуществляется планирование, проведение и обработка серии имитационных экспериментов, что обеспечивает возможность: во-первых, полностью исключить интуитивный подход в имитационном моделировании, заменив его научно обоснованной программой проведения экспериментального исследования, включающей объективную оценку результатов эксперимента на всех этапах исследования; во-вторых, оперативно и при минимально необходимом объеме затрачиваемых ресурсов получать многочисленные регрессионные модели, ориентированные на различные совокупности входных факторов и объективно и всесторонне отражающие специфику исследуемых бизнес-процессов в контексте разнообразных аспектов; в-третьих, полученные математические зависимости могут быть использованы как для оптимизации существующих бизнес-процессов, так и для тестирования новых, ещё не опробованных на практике, бизнес-решений.

\section{Список литературы}

1. Алексеев А.О., Бутко А.О., Колесников Д.А. Оптимизация бизнеспроцессов при помощи имитационного моделирования // Информационные технологии в проектировании и производстве. 2019. № 1 (173). С. 13-19.

2. Большаков И.Н. Имитационное моделирование бизнес-процессов и экономических систем // Международная конференция по мягким вычислениям и измерениям. 2018. Т. 2. С. 715-718.

3. Кирчева Е.М., Осенний В.В., Манютина С.Ю. Современные методы анализа бизнес-процессов с применением имитационного моделирования // Энигма. 2019. Т. 1. № 16-1. С. 109-122.

4. Круликовский А.П., Антропова А.А. Имитационное моделирование 
бизнес-процессов // Труды XIV Международной научно-практической конференции «Теория и практика экономики и предпринимательства». Симферополь, 2017. С. 191-192.

5. Михайлова С.Е., Сасева А.В., Пучков А.Ю. Обзор программных средств имитационного моделирования экономических процессов // Сборник научных трудов Международной научно-практической конференции «Кластерные инициативы в формировании прогрессивной структуры национальной экономики». Курск, 2015. С. 230-232.

6. Рассказов В.А., Касперский Ю.С. Инструментальные средства имитационного моделирования бизнес-процессов // Материалы $\mathrm{X}$ Международной научно-практической конференции «Государство и бизнес. Современные проблемы экономики». СПб., 2018. C. $132-137$.

7. Сиротин Д.В. Моделирование развития экономики региона в условиях Индустрии 4.0 // Наука Красноярья. 2019. Т. 8. № 3. С. 98-108.

8. Скородумов П.В. Имитационное моделирование экономических систем: программные средства и направления их совершенствования // Проблемы развития территории. 2015. № 2 (76). С. 62-72.

9. Скрипник Д.В., Яхонтова И.М. Современные подходы к имитационному моделированию бизнес-процессов // Сборник материалов VIII международного форума «Информационное общество: современное состояние и перспективы развития». Краснодар, 2017. С. 83-85.

10. Сытченко Д.Ю., Брежнев А.В. Программное обеспечение имитационного моделирования: языки и предметно-ориентированные программы моделирования // Сборник материалов Всероссийской конференции «Программная инженерия: современные тенденции развития и применения». Курск, 2017. С. 164-169.

11. Barad M. Design of Experiments (DOE) - A Valuable Multi-Purpose Methodology // Applied Mathematics. 2014. No. 5, pp. 2120-2129.

12. Durakovic B. Design of Experiments Application, Concepts, Examples: State of the Art // Periodicals of Engineering and Natural Sciences. 2017. Vol. 5. No. 3, pp. 421-439.

13. Grömping U. R Package DoE.base for Factorial Experiments // Journal of Statistical Software. 2018. Vol. 85. Issue 5, pp. 1-41. 
14. Montgomery D.C. Design and Analysis of Experiments. New York, 2013. $684 \mathrm{p}$.

15. Rüttimann B.G., Wegener K. The Power of DOE: How to Increase Experimental Design Success and Avoid Pitfalls // Journal of Service Science and Management. 2015. No. 8, pp. 250-258.

\section{References}

1. Alekseev A.O., Butko A.O., Kolesnikov D.A. Optimizatsiya biznes-protsessov pri pomoshchi imitatsionnogo modelirovaniya [Optimization of business processes using simulation modeling]. Informatsionnye tekhnologii v proektirovanii i proizvodstve. 2019. No. 1 (173), pp. 13-19.

2. Bol'shakov I.N. Imitatsionnoe modelirovanie biznes-protsessov i ekonomicheskikh system [Simulation modeling of business processes and economic systems]. Mezhdunarodnaya konferentsiya po myagkim vychisleniyam i izmereniyam. 2018. Vol. 2, pp. 715-718.

3. Kircheva E.M., Osenniy V.V., Manyutina S.Yu. Sovremennye metody analiza biznes-protsessov s primeneniem imitatsionnogo modelirovaniya [Modern methods of business process analysis using simulation modeling]. Enigma. 2019. Vol. 1. No. 16-1, pp. 109-122.

4. Krulikovskiy A.P., Antropova A.A. Imitatsionnoe modelirovanie biznes-protsessov [Simulation modeling of business processes]. Trudy XIV Mezhdunarodnoy nauchno-prakticheskoy konferentsii «Teoriya i praktika ekonomiki i predprinimatel'stva» [Proceedings of the XIV International Scientific and Practical Conference "Theory and Practice of Economics and Entrepreneurship]. Simferopol', 2017, pp. 191-192.

5. Mikhaylova S.E., Saseva A.V., Puchkov A.Yu. Obzor programmnykh sredstv imitatsionnogo modelirovaniya ekonomicheskikh protsessov [Overview of software tools for simulation modeling of economic processes]. Sbornik nauchnykh trudov Mezhdunarodnoy nauchno-prakticheskoy konferentsii «Klasternye initsiativy v formirovanii progressivnoy struktury natsional'noy ekonomiki» [Collection of scientific papers of the International scientific-practical conference "Cluster initiatives in the formation of a progressive structure of the national economy"]. Kursk, 2015, pp. 230-232. 
6. Rasskazov V.A., Kasperskiy Yu.S. Instrumental'nye sredstva imita-tsionnogo modelirovaniya biznes-protsessov [Software tools for simulation modeling of business processes]. Materialy X Mezhdunarodnoy nauchno-prakticheskoy konferentsii «Gosudarstvo i biznes. Sovremennye problemy ekonomiki» [Materials of the X International Scientific and Practical Conference "State and Business. Modern problems of the economy"]. SPb., 2018, pp. 132-137.

7. Sirotin D.V. Modelirovanie razvitiya ekonomiki regiona v usloviyakh Industrii 4.0 [Development modeling of the regional economy in the industry 4.0 conditions]. Nauka Krasnoyar'ya. 2019. Vol. 8. No. 3, pp. 98-108.

8. Skorodumov P.V. Imitatsionnoe modelirovanie ekonomicheskikh sistem: programmnye sredstva i napravleniya ikh sovershenstvovaniya [Simulation modeling of economic systems: software tools and areas of improvement]. Problemy razvitiya territorii. 2015. No. 2 (76), pp. 62-72.

9. Skripnik D.V., Yakhontova I.M. Sovremennye podkhody k imitatsion-nomu modelirovaniyu biznes-protsessov [Modern approaches to simulation modeling of business processes]. Sbornik materialov VIII mezhdunarodnogo foruma "Informatsionnoe obshchestvo: sovremennoe sostoyanie i perspektivy razvitiya» [Proceedings of the VIII international forum "Information Society: current status and development prospects"]. Krasnodar, 2017, pp. 83-85.

10. Sytchenko D.Yu., Brezhnev A.V. Programmnoe obespechenie imitatsion-nogo modelirovaniya: yazyki i predmetno-orientirovannye programmy modeli-rovaniya [Simulation software: languages and object-oriented modeling programs]. Sbornik materialov Vserossiyskoy konferentsii «Programmnaya inzheneriya: sovremennye tendentsii razvitiya i primeneniya" [Proceedings of the All-Russian Conference "Software Engineering: Current Trends of Development and Application"]. Kursk, 2017, pp. 164-169.

11. Barad M. Design of Experiments (DOE) - A Valuable Multi-Purpose Methodology. Applied Mathematics. 2014. No. 5, pp. 2120-2129.

12. Durakovic B. Design of Experiments Application, Concepts, Examples: State of the Art. Periodicals of Engineering and Natural Sciences. 2017. Vol. 5. No. 3, pp. 421-439. 
13. Grömping U. R Package DoE.base for Factorial Experiments. Journal of Statistical Software. 2018. Vol. 85. Issue 5, pp. 1-41.

14. Montgomery D.C. Design and Analysis of Experiments. New York, 2013. $684 \mathrm{p}$.

15. Rüttimann B.G., Wegener K. The Power of DOE: How to Increase Experimental Design Success and Avoid Pitfalls. Journal of Service Science and Management. 2015. No. 8, pp. 250-258.

\section{ДАННЫЕ ОБ АВТОРЕ}

Тюльпинова Нина Владимировна, доцент кафедры «Технология машиностроения», кандидат технических наук, доцент Брянский государственный технический университет бульвар 50 лет Октября, 7, г. Брянск, 241035, Российская Федерация ninatulpinova@mail.ru

\section{DATA ABOUT THE AUTHOR}

Tyulpinova Nina Vladimirovna, Associate Professor «Manufacturing engineering», Candidate of Engineering Sciences, Associate Professor

Bryansk State Technical University

7, 50 let Octyabrya Blvd, Bryansk, 241035, Russian Federation ninatulpinova@mail.ru

ORCID: 0000-0002-1485-7238 